\title{
Validating a Plasma Momentum Flux Sensor to an Inverted Pendulum Thrust Stand
}

\author{
Benjamin W. Longmier* \\ University of Houston, Houston, Texas 77204 \\ Bryan M. Reid产 and Alec D. Gallimore \\ University of Michigan, Ann Arbor, Michigan 48109 \\ Franklin R. Chang-Díaz, $\underline{\underline{\Xi}}$ Jared P. Squire, $\mathbb{I}$ and Tim W. Glover** \\ Ad Astra Rocket Company, Webster, Texas 77598 \\ Greg Chavers辛 \\ NASA Marshall Space Flight Center, Huntsville, Alabama 35812 \\ and \\ Edgar A. Bering III乼 \\ University of Houston, Houston, Texas 77204 \\ DOI: $10.2514 / 1.35706$
}

\begin{abstract}
The accuracy of a plasma impact force sensor was compared with that of the more commonly used inverted pendulum thrust stand using a $5 \mathrm{~kW}$ Xe Hall effect thruster. An improved plasma momentum flux sensor was designed and constructed based on a previous design. Real-time force measurements were made with both the plasma momentum flux sensor and the inverted pendulum thrust stand. The plasma momentum flux sensor measured the force exerted onto it by the Hall effect thruster exhaust plume with a resolution of $0.1 \mathrm{mN}$ and an average discrepancy of $2 \%$ compared with thrust stand measurements. Experiments were completed using a $9 \mathrm{~m}$ by $6 \mathrm{~m}$ cylindrical vacuum chamber. The total force from the Hall effect thruster was modulated from 34 to $356 \mathrm{mN}$ by varying both the anode voltage, from 150 to $500 \mathrm{~V}$, and the neutral Xe gas flow rate, from 5 to $15 \mathrm{mg} / \mathrm{s}$.
\end{abstract}

\section{Introduction}

$\mathbf{I}_{\mathrm{T}}^{\mathrm{t}}$ T IS often physically or financially impractical to measure the force produced by an electric thruster using the traditional technique of mounting the thruster on an inverted pendulum thrust stand. Instead, a simple, robust, and low-cost plasma impact force sensor can be used to reliably determine the total force produced by an electric propulsion thruster. Until now, only a handful [1-5] of experiments focused on impact force sensors. Virtually no experiments have attempted to compare the accuracy of an impact thrust sensor to the more commonly used inverted pendulum thrust stand. Experimental tests were completed using the P5 thruster [6-8] ], a $5 \mathrm{~kW}$ Xe Hall effect thruster (HET), and demonstrated that the thrust from the P5 inferred from measurements made with a plasma

Presented as Paper 4739 at the 44th AIAA/ASME/SAE/ASEE Joint Propulsion Conference \& Exhibit, Hartford, CT, 21-23 July 2008; received 18 June 2008; revision received 21 December 2008; accepted for publication 4 January 2009. Copyright (C) 2009 by the American Institute of Aeronautics and Astronautics, Inc. All rights reserved. Copies of this paper may be made for personal or internal use, on condition that the copier pay the $\$ 10.00$ percopy fee to the Copyright Clearance Center, Inc., 222 Rosewood Drive, Danvers, MA 01923; include the code 0748-4658/09 $\$ 10.00$ in correspondence with the CCC.

*Postdoctoral Fellow, Physics Department, 141 West Bay Area Boulevard. Member AIAA.

${ }^{\dagger}$ Ph.D. Candidate, Aerospace Engineering, University of Michigan, 1919 Green Road, Room B107. Member AIAA.

Frofessor of Aerospace Engineering and of Applied Physics, Associate Dean for Academic Programs and Initiatives, Aerospace Engineering, 1919 Green Road, Room B107. Associate Fellow AIAA.

${ }^{\S}$ Chief Executive Officer, Ad Astra Rocket Company, 141 West Bay Area Boulevard. Associate Fellow AIAA.

"Director of Research, Ad Astra Rocket Company, 141 West Bay Area Boulevard. Member AIAA.

${ }^{* *}$ Director of Development, Ad Astra Rocket Company, 141 West Bay Area Boulevard. Member AIAA.

${ }^{\dagger}$ Aerospace Engineer, Mission Operations Laboratory. Member AIAA.

\$Professor, Physics and Electrical \& Computer Engineering, 617 Science $\&$ Research Building 1. Associate Fellow AIAA. momentum force sensor (PMFS) agree very closely with the thrust measurements made by a conventional inverted pendulum thrust stand.

The PMFS described in this paper was designed and constructed based on a previous design [1]. The PMFS was placed in the flowing plasma stream of the $\overline{\mathrm{P}} 5 \mathrm{HET}$, for which real-time force measurements were made with both the PMFS and the thrust stand. The PMFS was able to measure the force exerted on it with a resolution of $0.1 \mathrm{mN}$ and an average discrepancy of $2 \%$ compared with the thrust stand. The total force from the HET was modulated from 34 to $356 \mathrm{mN}$ by varying both the anode voltage, from 150 to $500 \mathrm{~V}$, and the neutral Xe gas flow rate, from 5 to $15 \mathrm{mg} / \mathrm{s}$. Additionally, the majority of the force data taken during the experiment was completed as a blind study in which force measurements from both techniques were disclosed only after the experiment was completed. Though the experiments show a high accuracy for HETs with ion energies ranging from 130 to $440 \mathrm{eV}$, the PMFS showed no indication of saturation with ion energy, plasma flux, local magnetic field strength, or force magnitude, indicating that the full range of the PMFS usefulness is still largely unexplored. The PMFS may find uses in many flowing plasma and electric propulsion applications for which an accurate, low-cost force sensor is required.

\section{Experimental Setup}

A. Chamber and Diagnostic Setup

The Large Vacuum Test Facility, a cylindrical stainless-steel-clad vacuum chamber at the University of Michigan-Ann Arbor, is a 9-mlong by 6-m-diam chamber used primarily for the testing of research and space-qualified electric propulsion thrusters [9]. The chamber is evacuated by two $2000 \mathrm{ft}^{3} / \mathrm{min}$ blowers and four $400 \mathrm{ft}^{3} / \mathrm{min}$ mechanical pumps to a rough vacuum level of 30-100 mtorr [9]. To reach high vacuum, $\sim 10^{-7}$ torr, the vacuum chamber employs seven CVI TM-1200 nude cryopumps, with a combined pumping speed of $500,000 \mathrm{l} / \mathrm{s}$ of air and 240, $000 \mathrm{l} / \mathrm{s}$ of xenon. However, because of the low flow rates of the P5 thruster, only four of the seven cryopumps were used during this experiment, with a resulting $\mathrm{Xe}$ 
pumping speed of 140,000 1/s. The four cryopumps yielded an average base vacuum pressure of $3 \times 10^{-7}$ torr and a chamber pressure of $4.8 \times 10^{-6}, 7.9 \times 10^{-6}$, and $1.1 \times 10^{-5}$ torr, with a combined thruster and cathode flow rate of 5,10, and $15 \mathrm{mg} / \mathrm{s} \mathrm{Xe}$, respectively.

The P5 thruster was mounted on an inverted pendulum thrust stand, described in detail by Haag [10]. The inverted pendulum thrust stand and, hence, the P5 thruster, was fixed in place within the vacuum chamber, and the PMFS was mounted on a two-axis translation stage within the vacuum chamber. The translation stage allowed the PMFS to scan a separation distance of $94-23 \mathrm{~cm}$ from the thruster exit plane and allowed for a lateral range of $100 \mathrm{~cm}: 70 \mathrm{~cm}$ from the axis in one direction and $30 \mathrm{~cm}$ from the axis in the other direction. For all force measurements, the graphite PMFS target was centered radially and placed at various axial separation distances. However, the translation stage moved through its entire lateral range of motion to obtain a radial ion flux profile with a Faraday probe biased into ion saturation. A 3-mm-diam tungsten rod enclosed in an alumina shroud served as the Faraday probe. The tip of the rod was flush with the alumina tube to help prevent a radial sheath expansion with biases more negative than the floating potential. The Faraday probe was mounted at the same vertical and axial position as the graphite PMFS target, but with a $20 \mathrm{~cm}$ radial offset from the PMFS target. The surface of the Faraday probe was also cleaned for $10 \mathrm{~min}$ via xenon ion bombardment before data collection.

To measure the ion energy in the plume, a retarding potential analyzer (RPA) was installed on a 60-cm-long boom that was able to sweep through the plume [11]. Measurements were then taken with the RPA located $58.5 \mathrm{~cm}$ from the exit plane of the thruster. The RPA was mounted on the boom structure so that it could be moved out of the thruster plume when PMFS measurements were taken. A small floating probe was also installed on the boom and was used to measure the plasma floating potential $58.5 \mathrm{~cm}$ from the exit plane of the thruster.

\section{B. Hall Thruster}

All data within this paper, with the exception of Sec. II.D, were taken using the P5 Xe HET, a $5 \mathrm{~kW}$ HET designed and used primarily for research purposes [5-8]. Previous data indicate that the P5 has performance characteristics comparable to commercial spacequalified $5 \mathrm{~kW}$ HETs [5-8]. The P5 has a discharge chamber outer diameter of $173 \mathrm{~mm}$ and channel width of $25 \mathrm{~mm}$. Although designed for steady-state operation at $5 \mathrm{~kW}$, the thruster was operated at power levels from $0.71(150,4.70)$ to $7.65 \mathrm{~kW}(500 \mathrm{~V}, 15.30 \mathrm{~A})$ during this experiment. The P5 used xenon gas as the propellant for the duration of this testing campaign and for all data presented in this paper.

\section{Concept and Construction of the PMFS}

The PMFS consists of a 9-cm-diam graphite target disc attached to a 10-cm-long insulating alumina rod. The stiff alumina rod then connects to a small titanium bar $(5.72 \times 1.30 \mathrm{~cm})$ on which a series of four high-output semiconductor strain gauges, Micron Instruments model number SS-090-060-1150P, are mounted between two holes on an "isthmus" on the titanium bar, as seen in Fig. 1. The isthmus acts as a stress concentrator and increases the sensitivity of the device. The strain gauges are connected electrically in a Wheatstone bridge configuration so that changes in temperature of the titanium bar do not affect the linearity of the strain gauge output.

When the electrically floating graphite disc is immersed in flowing plasma (e.g., the plume of a HET), the force from the plasma impacting the graphite target is translated into a strain in the titanium beam through a moment arm equal to the length of the alumina rod plus the clamp length. A small graphite shield was also used to keep the entire titanium bar and strain gauge assembly shielded from the flowing plasma and associated thermal and electrical noise.

The resolution of the PMFS and the inverted pendulum thrust stand described in this paper was 0.1 and $0.5 \mathrm{mN}$, respectively, which allowed for sufficiently sensitive measurements of the force applied

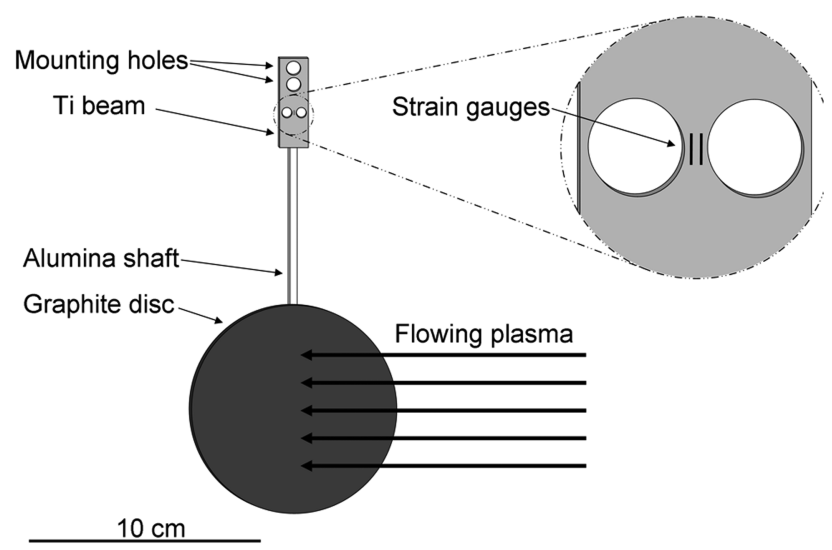

a)

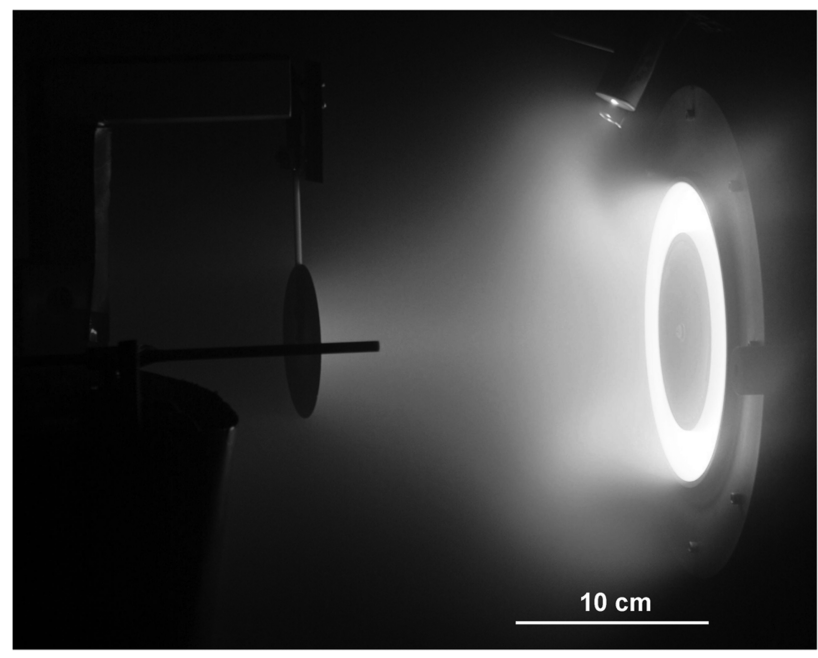

b)

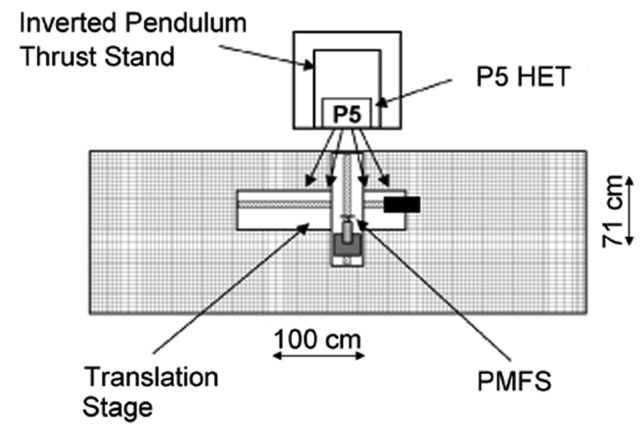

c)

Fig. 1 Shown are the following: a) schematic of the PMFS assembly and magnification of strain gauge arrangement mounted on the $\mathbf{T i}$ isthmus, b) P5 HET and PMFS during the closest approach (the Faraday probe biased into ion saturation can be seen in the foreground and the PMFS graphite target disc in the background), and c) overhead schematic of the the P5 HET and the force target.

by the exhaust plasma. The typical error associated with the inverted pendulum thrust stand is $\pm 2 \mathrm{mN}$ for a measured force of $100 \mathrm{mN}$.

If an increased PMFS force resolution were required, the length of the alumina moment arm could be increased, acting to increase the output from the strain gauges for a particular force applied to the graphite target. However, increasing the arm length of the device also decreases the resonant frequency response of the device. This limitation is generally not a concern for steady-state thruster operation. If the thruster (or some other source of flowing plasma) were operated in a pulsed mode, then data analysis is simplified if the moment arm was selected such that the natural period of the PMFS device is much shorter than the thruster pulse duration. 
The graphite target only measured a portion of the total axial force generated by the HET in each measurement. The PMFS target diameter was $52 \%$ of the P5 thruster channel o.d. An azimuthally integrated radial profile of the ion flux was used to account for the portion of the plasma plume that was not intercepted by the graphite target. For each force measurement presented in this paper, a corresponding radial profile of the ion flux was collected by a Faraday probe and used to determine the total axial force produced by the thruster. Figure 2 is a representative radial ion flux profile from the P5 HET plume; in this case, the graphite target was $50 \mathrm{~cm}$ downstream from the exit plane of the thruster. The ion flux was recorded with a tungsten electrode that was recessed inside of an alumina tube and was biased at $-14.95 \mathrm{~V}$, roughly $3 \mathrm{Te}$ lower in voltage than the plasma potential, but not too low so as to create additional ionization in the far ion saturation region. A high input impedance data logger was used to record the current through a $107.3 \Omega$ shunt resistor.

The ratio of the total axial ion flux $(r=0-100 \mathrm{~cm})$, numerically integrated over the entire plume assuming cylindrical symmetry, to that of the axial ion flux intercepted by the graphite target $(r=0-4.5 \mathrm{~cm})$ is given by

$$
\frac{\sum_{x=0}^{x=1000} \pi\left(r_{x+1}^{2}-r_{x}^{2}\right) I\left(r_{x}\right)}{\sum_{x=0}^{x=45} \pi\left(r_{x+1}^{2}-r_{x}^{2}\right) I\left(r_{x}\right)}
$$

where $I\left(r_{x}\right)$ is the ion current as measured by a Faraday probe biased into ion saturation at a radius $r_{x}$ in the plasma exhaust. Here, $x$ ranges from 0 to 1000 for $r_{x}$ values from 0 to $100 \mathrm{~cm}$. Thrust contributions from the plume at radii greater than $100 \mathrm{~cm}$, the maximum range of the translation stage, are negligible for the data presented in this paper.

The total axial force, $F_{\text {Total }}$, produced by the HET is determined by multiplying the force measured by the graphite target, $F_{\text {Target }}$, by Eq. (1), which becomes

$$
F_{\text {Total }}=F_{\text {Target }} \frac{\sum_{x=0}^{x=1000} \pi\left(r_{x+1}^{2}-r_{x}^{2}\right) I\left(r_{x}\right)}{\sum_{x=0}^{x=45} \pi\left(r_{x+1}^{2}-r_{x}^{2}\right) I\left(r_{x}\right)}
$$

The assumption that the thruster plume is symmetric in the azimuthal direction leads to the largest source of error with the PMFS device. The asymmetries observed in the plume are likely due to nonuniform propellant delivery as the flow rate increased beyond its nominal design point of $10 \mathrm{mg} / \mathrm{s}$. A similar result was reported where fabrication error in the anode resulted in an asymmetric plume at high flow rates [9]. One way to reduce this error is to construct a two-dimensional map of the ion flux profile; however, this mapping was not performed due to the limitations of the translation stage.

Once a total force measurement was numerically integrated from the PMFS measurement and ion flux profile, momentum reflection and sputtering were taken into consideration and corrections were made, as described in Sec. IV.A.

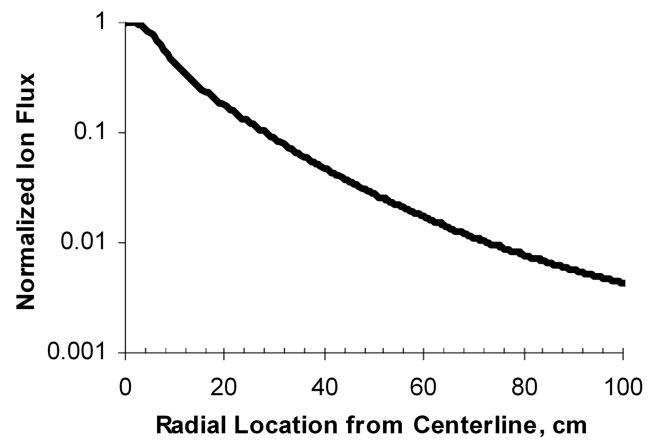

Fig. 2 A representative radial profile of the ion flux in the HET plume $50 \mathrm{~cm}$ downstream from the exit plane of the thruster using $15 \mathrm{mg} / \mathrm{s} \mathrm{Xe}$, $150 \mathrm{~V}$, and $16.6 \mathrm{~A}$.

\section{Calibration}

After the PMFS was installed in a rigid location within the vacuum chamber, in this case on a two-axis translation stage, and before the vacuum pump down, the PMFS was calibrated to find an accurate relationship between the output of the strain gauges and the force applied to the target. Calibration was performed in the same manner as was previously described by Chavers and Chang-Díaz [1], in which a set of calibrated weights were hung from a tethered string assembly to apply a known tension force on the graphite target disc. Figure $\underline{3}$ shows a representative example of the force-to-voltage relation for the particular device used in this experiment. The strain sensors had an output voltage-to-force relation of $0.64 \pm$ $0.01 \mathrm{mV} / \mathrm{mN}$ (or $1.56 \mathrm{mN} / \mathrm{mV}$ ). If the PMFS was correctly leveled within the vacuum chamber before testing, the resulting force per unit voltage line would intercept the origin, as depicted in Fig. 3 .

The data from Fig. 1 also indicate that the strain gauges are linear over a force range from 0 to at least $50 \mathrm{mN}$ for this particular device. Because the graphite target did not intercept all of the HET exhaust plume, this translates into a useful measurable P5 thruster force range of at least $0-1000 \mathrm{mN}$ for thruster-to-target separation distances between 23 and $94 \mathrm{~cm}$. A PMFS calibration was performed before and after every vacuum chamber pump down and venting to verify that the strain gauges did not exhibit a drift or creep in time. During the PMFS HET experiments, both PMFS calibrations agreed to within $0.3 \%$.

\section{Experimental Demonstration}

\section{A. Thruster-Sensor Separation Distance}

An initial thruster-to-target separation distance experiment was performed to determine which separation distances could be tolerated without significantly altering the performance of the thruster. The separation distance was decreased from 94 to $23 \mathrm{~cm}$ in roughly $10 \mathrm{~cm}$ increments. For each thrust measurement, the thruster was turned on and allowed to stabilize. Then, a radial ion flux profile was taken with the Faraday probe and a relative thrust measurement was taken with both techniques. The thruster was then turned off, and a baseline thrust measurement was taken, also with both techniques. The difference in signal strength between the thrust-on and thrust-off values was used to determine a thruster force. The slope from the respective PMFS and thrust stand calibration curves (i.e., $\mathrm{mN} / \mathrm{mV}$ ) was then used to determine an absolute force value from the relative force measurements. In this way, simultaneous force measurements could be made with both techniques. This procedure also helped to compensate for any drift in thruster performance or force over time.

An increase in the measured force was registered with both techniques when the PMFS disc approached the HET, as seen in Fig. 4. The PMFS data presented in Fig. 4 and Table 1 also include corrections for sputtering based on Stopping and Rañge of Ions in Matter/Transport of Ions in Matter (SRIM)/TRIM models [12] (see Sec. IV.A for further sputtering correction details).

The increase in thruster force with reduced thruster-target separation distance was previously observed by Chavers and Chang-Díaz [1] in a similar experiment and is attributed to an increased neutral pressure near the HET exit plane. Because of finite facility pressure, background neutrals continually reach the thruster

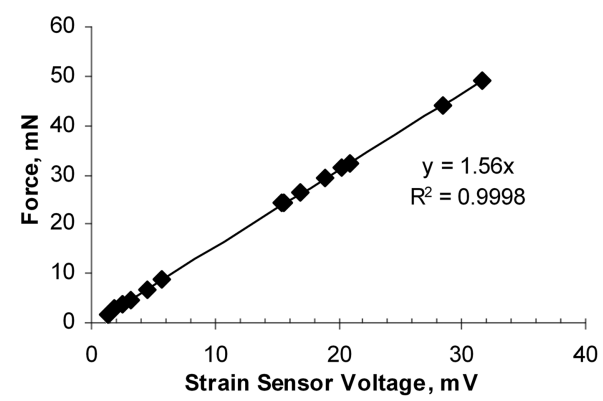

Fig. 3 A calibration graph used to convert strain sensor voltage to an applied force on the target. 


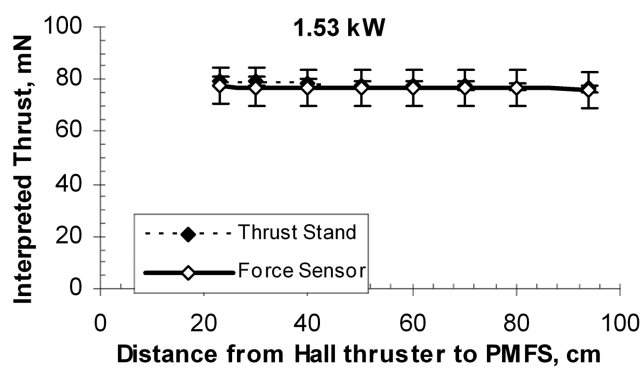

Fig. 4 Measured thrust vs the separation distance of the PMFS target and the HET. The thruster was operated at $1.53 \mathrm{~kW}$ using $5 \mathrm{mg} / \mathrm{s} \mathrm{Xe}$, $300 \mathrm{~V}$, and $5.1 \mathrm{~A}$.

discharge channel, become ionized, accelerated, and artificially increase thrust [13]. As the PMFS approaches the thruster exit plane, the neutral ingestion is expected to increase because of two effects. First, a fraction of the nonionized neutrals from the thruster will be reflected from the paddle surface with an isotropic distribution. Second, ions will impact the paddle and recombine at the surface to produce an additional isotropic reflected neutral flux. A fraction of these neutralized ions will also reach the thruster and artificially increase the thrust produced by the thruster. The HET was operated at $1.53 \mathrm{~kW}$, producing $5.1 \mathrm{~A}$ of discharge current with an anode bias of $300 \mathrm{~V}$, using a neutral Xe flow rate of $5 \mathrm{mg} / \mathrm{s}$. The average difference between the force measurements for the inverted pendulum thrust stand and PMFS techniques is $1.4 \%$ for this data set, which is considerably less than the error associated with either the PMFS or the inverted pendulum thrust stand for this set of experiments.

\section{B. Blind Study}

The following data from the thrust stand and the PMFS were taken and analyzed as a "blind study." That is, the forces measured by the thrust stand and the PMFS were recorded separately and were only disclosed to each respective research team three weeks later, after a complete calibration and sputter analysis had been performed. The PMFS was positioned on the thruster centerline $50 \mathrm{~cm}$ from the exit plane of the thruster while the Xe flow rate and the thruster anode voltage were altered to achieve a range of ion energy, ion flux, and total force. Figure $\underline{5}$ shows the thrust from the thrust stand and the PMFS measurements as a function of the calculated HET power. Table 2 shows the numeric results displayed in Fig. 5.

The large discrepancies associated with the 7.65, 4.38, and $0.71 \mathrm{~kW}$ power levels were later determined to be due to an incorrect scaling value used with the radial ion flux traces for the PMFS. This

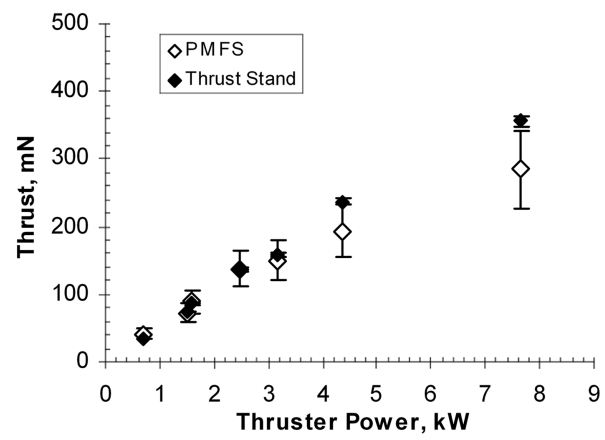

Fig. 5 Blind study thrust measurements for a variety of P5 HET power levels. The PMFS was located $50 \mathrm{~cm}$ downstream from the thruster exit plane.

issue was corrected after the blind study and the corrected data are presented in Sec. III.C.

\section{Corrections to Blind Study}

After both sets of force data from the blind study were exchanged between research teams at the University of Michigan and the University of Houston, it was discovered that three of the PMFS data points fell much farther away from the inverted pendulum data points. In a post-blind-study analysis, it was discovered that an incorrect centerline was used for three of the radial ion flux traces, throwing off the total force value for the three PMFS measurements with a larger discrepancy. Figure 6 shows a graph of the radial ion flux profiles for several thruster gas flow rates and anode voltage levels with the correct thruster centerline superimposed on the graph.

During the initial data analysis, an incorrect plume centerline was chosen based on the two largest peaks and the smallest peak in Fig. 6, instead of the true plume centerline. This incorrect centerline was used to calculate the force values for the two largest peaks and the smallest peak, resulting in three incorrect force values. The remaining ion flux peaks were analyzed separately with the correct thruster centerline. The clearer bimodal nature of the remaining peaks led to the correct choice of the thruster plume centerline and the correct calculation of force values for the PMFS. The updated and correct results are shown in Fig. 7a and Table 3. A more accurate centerline for all of the peaks, found by fitting a bimodal distribution to the data in Fig. 6 , is located at a radial position of $0 \mathrm{~cm}$ and shown in Fig. 6. The Faraday probe that was mounted on the translation stage had a radial range of $138.1 \mathrm{~cm}$.

After the correct centerline was used to calculate the total thrust with the PMFS technique, the three previously errant force measurements agreed much more closely with the inverted pendulum

Table 1 Measured thrust vs the separation distance of the PMFS target from the HET

\begin{tabular}{lcccccccc}
\hline \hline Separation distance, cm & 94 & 80 & 70 & 60 & 50 & 40 & 30 & 23 \\
PMFS, $m N$ & 76.1 & 76.6 & 76.8 & 76.8 & 76.9 & 76.9 & 77.1 & 77.3 \\
Thrust stand, $m N$ & 76.3 & 77.1 & 77.6 & 77.9 & 77.9 & 78.4 & 79.2 & 79.2 \\
Difference, \% & 0.3 & 0.6 & 1.1 & 1.4 & 1.2 & 1.9 & 2.6 & 2.4 \\
Anode Voltage, V & 300 & 300 & 300 & 300 & 300 & 300 & 300 & 300 \\
Xe flow rate, mg/s & 5 & 5 & 5 & 5 & 5 & 5 & 5 & 5 \\
Anode current, A & 5.1 & 5.1 & 5.1 & 5.1 & 5.1 & 5.1 & 5.1 & 5.1 \\
\hline \hline
\end{tabular}

Table 2 Measured thrust for a variety of P5 HET power levels. The PMFS was located $50 \mathrm{~cm}$ downstream from the thruster exit plane

\begin{tabular}{lccccccc}
\hline \hline Thruster power, kW & 0.71 & 1.50 & 1.58 & 2.49 & 3.15 & 4.38 & 7.65 \\
PMFS, $m N$ & 41.4 & 72.7 & 89.2 & 138.2 & 149.7 & 193 & 284.3 \\
Thrust stand, $m N$ & 33.9 & 73.6 & 85.9 & 136.6 & 158.8 & 237.1 & 355.8 \\
Difference, \% & 22.3 & 1.2 & 3.8 & 1.2 & 5.7 & 18.6 & 20.1 \\
Anode Voltage, V & 150 & 300 & 150 & 150 & 300 & 300 & 500 \\
Xe flow rate, mg/s & 5 & 5 & 10 & 15 & 10 & 15 & 15 \\
Anode current, A & 4.7 & 5.0 & 10.5 & 16.6 & 10.5 & 14.6 & 15.3 \\
\hline \hline
\end{tabular}




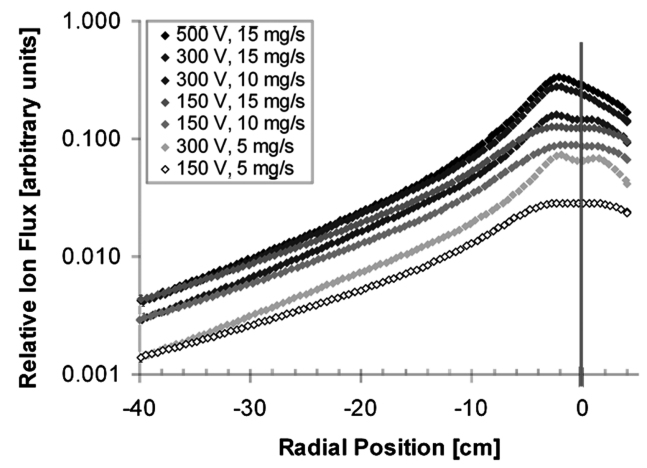

Fig. 6 A portion of the relative radial ion flux profiles for several thruster gas flow rates and anode voltage levels. The PMFS and the Faraday probe were located $50 \mathrm{~cm}$ downstream from the thruster exit plane. The vertical line shows the correct plume centerline.

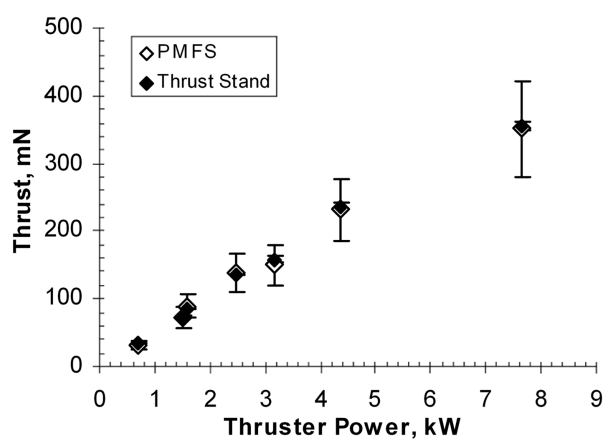

a)

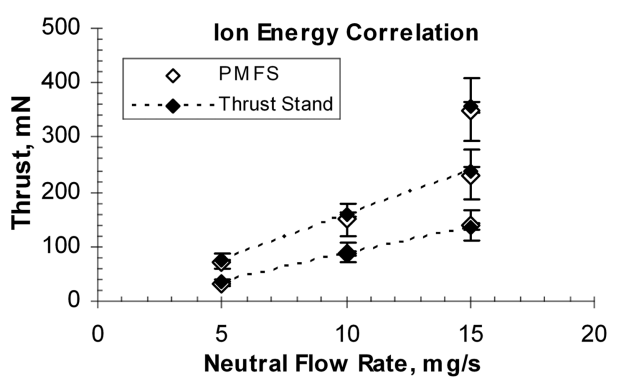

b)

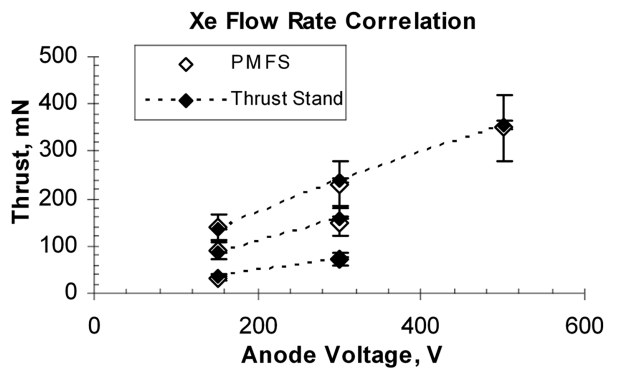

c)

Fig. 7 Corrected blind study thrust measurements for a variety of P5 HET power levels. The PMFS was located $50 \mathrm{~cm}$ downstream from the thruster exit plane.

thrust values. After using the correct plume centerline, the largest difference between the thrust stand measurements and the PMFS measurements was $5.7 \%$, with an average discrepancy for this data set of $2.8 \%$, as seen in Table 3 .

In an effort to identify a trend in the PMFS accuracy as a function of ion energy and neutral gas flow rate, the relevant PMFS and thrust stand force data are graphed as a function of the thruster neutral flow rate, Fig. $7 \mathrm{~b}$, and as a function of the thruster anode voltage, Fig. $7 \mathrm{c}$. No clear correlation of PMFS accuracy is obvious as the anode voltage or neutral gas flow rate are varied. To the first order, the discrepancy between the PMFS and the thrust stand appears to be largely unaffected by the HET system parameters. However, data from Fig. 4 do indicate a discernible trend, with a larger discrepancy between the two force measurement techniques as the PMFS approaches the thruster exit plane from a separation distance of 94 $23 \mathrm{~cm}$.

The PMFS has proven to be accurate compared with the inverted pendulum thrust stand with a maximum observed difference of $5.7 \%$ over a large range of applied force, ion energy, and neutral gas flow settings. The PMFS should find considerable application in the lowthrust plasma community due to its simplicity, robustness, and verified accuracy.

\section{Momentum Flux Parameters}

\section{A. Surface Sputtering}

Target material choice for the PMFS is a nontrivial task owing to the bombardment from high-energy ions in the exhaust plume of the HET. At a maximum operating power level, the P5 HET was able to produce incident ions with an average energy of approximately $440 \mathrm{eV}$. This ion energy is far beyond the sputter threshold of any

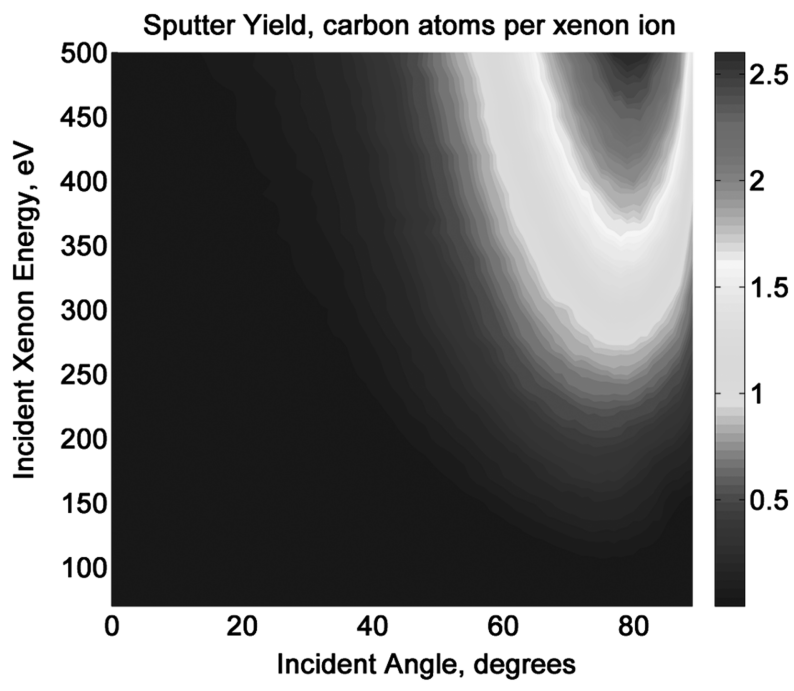

a)

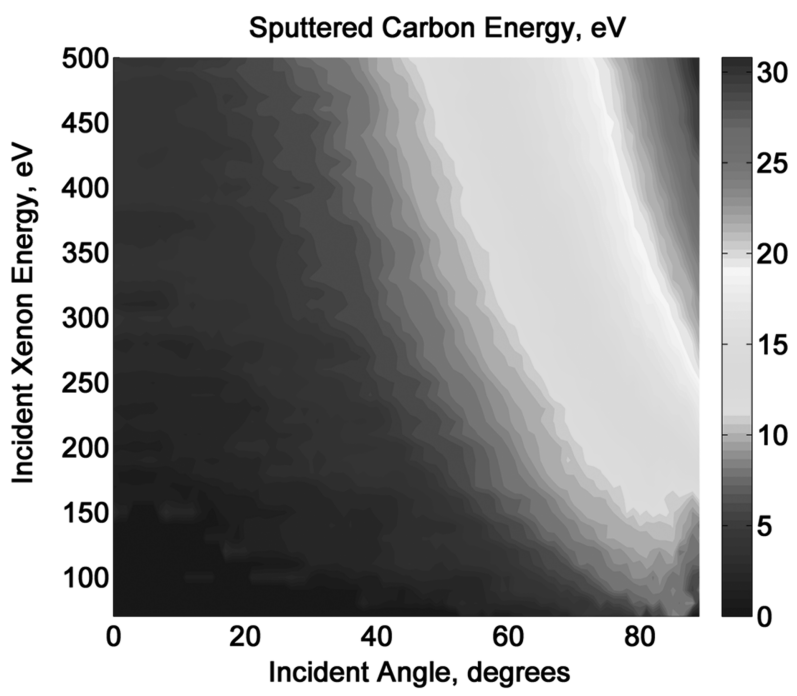

b)

Fig. 8 Shown are a) carbon sputter yield ( $\mathrm{C}$ atom released per incident $\mathrm{Xe}$ ion) and $\mathrm{b}$ ) average sputtered $\mathrm{C}$ atom energy as a function of incident angle from the target perpendicular for various incident $\mathrm{Xe}$ ion energies. 
Table 3 Measured thrust for a variety of P5 HET power levels. The PMFS was located $50 \mathrm{~cm}$ downstream from the thruster exit plane

\begin{tabular}{lccccccc}
\hline \hline Thruster power, kW & 0.71 & 1.50 & 1.58 & 2.49 & 3.15 & 4.38 & 7.65 \\
PMFS, $m N$ & 32.6 & 72.7 & 89.2 & 138.2 & 149.7 & 231.4 & 350.7 \\
Thrust stand, $m N$ & 33.9 & 73.6 & 85.9 & 136.6 & 158.8 & 237.1 & 355.8 \\
Difference, \% & 3.6 & 1.2 & 3.8 & 1.2 & 5.7 & 2.4 & 1.4 \\
Anode Voltage, V & 150 & 300 & 150 & 150 & 300 & 300 & 500 \\
Xe flow rate, mg/s & 5 & 5 & 10 & 15 & 10 & 15 & 15 \\
Anode current, A & 4.7 & 5.0 & 10.5 & 16.6 & 10.5 & 14.6 & 15.3 \\
\hline \hline
\end{tabular}

material. One simply tries to reduce the sputtering yield to reduce the associated momentum corrections and to reduce contamination of the HET from sputtered target material. With an $\mathrm{Ar}, \mathrm{Kr}$, or $\mathrm{Xe}$ propellant, the natural target material choice for low sputter yield is graphite. This low yield is in part a result of the covalent $\mathrm{C}-\mathrm{C}$ bond strength, which results in the lowest sputter yield compared with any other material (with the exception of diamond) when the incident particles are more than twice as massive as the target atom [14]. Experiments have also shown that, for incident Xe ions, graphite has the lowest sputtering yield (with the exception of diamond) [15]. To quantify sputtering yield rates in this experiment, an RPA was used to measure the incident ion energy distribution from the HET for every force measurement data point. For typical operation, the P5 HET produces ions with an average energy equal to $50 \mathrm{~V}$ less than the anode voltage. That is, $\mathrm{Xe}$ ions with an average energy of $250 \mathrm{eV}$ are observed from an anode setting of $300 \mathrm{~V}$ with the P5 HET [11].

The sputtering yield from a target surface is also highly dependent on the incident angle of the incoming particle, where incident particle trajectories that are normal to the surface generally produce lower sputtering yields and incident particle trajectories that are $\sim 70-85 \mathrm{deg}$ from normal have the largest sputtering yields. Figures $8 \mathrm{a}$ and $8 \mathrm{~b}$ show the SRIM code [10] simulations for graphite sputter yield and energy per sputtered carbon atom as a function of incident angle from normal for a range of incident Xe ion energy.

In Figs. $8 \mathrm{a}$ and $8 \mathrm{~b}$, a Xe particle with an incident angle of $0 \mathrm{deg}$ means the particle is perpendicular (normal) to the surface.

SRIM sputter yield and sputtered particle energy simulations were completed once the average incident Xe ion energy was determined from RPA data and the possible incident ion trajectories were determined from the thruster-to-target separation distance and thruster dimensions. The SRIM simulations gave the yield and energy of sputtered $\mathrm{C}$ atoms. These results were used to find the added momentum flux on the target caused by the exiting sputtered particles. The sputter simulation output is presented in Tables $\underline{4}$ and $\underline{5}$ for the separation distance experiment and the corrected "blind study" experiment, respectively. The last row in Tables 4 and 5 shows the momentum change on the PMFS force target that was caused by sputtered $\mathrm{C}$ atoms leaving the target surface.

Because the sputter yield varies with incident particle angle, an integrated value was computed based on the probable incident ion trajectories from the HET channel impacting an annular section of the circular PMFS target. Ion energy values were measured with an RPA at the $53.5 \mathrm{~cm}$ location and were used for all sputter calculations with the local ion flux measurements from each location. These are presented in Tables $\underline{4}$ and $\underline{5}$ as integrated sputter yield.

Simulations from ${ }^{-}$SRIM show that the Xe ions with an energy between 8 and $500 \mathrm{eV}$ implant themselves $1-4 \mathrm{~nm}, 10-40$ times the atomic spacing for carbon atoms, into the surface of the graphite target in an inelastic collision and do not immediately "bounce" off of the target surface in an elastic collision. For Xe particles impacting a graphite surface, these inelastic implantations occur for Xe particle energies above $\sim 8 \mathrm{eV}$, roughly the $\mathrm{C}-\mathrm{C}$ surface binding energy for graphite. This inelastic ion implantation claim is supported by the experimental force comparison results in Secs. III.A and III.C.

According to the SRIM simulations, the increase in measured momentum from carbon atoms sputtered off of the PMFS graphite target is, at most, $1.3 \%$ of the total measured momentum from the incident Xe ions. This small correction is included in all of the measurements presented in this paper, but is typically much less than the other errors in the measurements.

Table 4 Carbon sputter yield, average sputtered $\mathrm{C}$ atom energy, and the associated momentum increase as a function of incident $\mathrm{Xe}$ angle and energy for various thruster-target separation distances

\begin{tabular}{lcccccccc}
\hline \hline Separation distance, cm & 94 & 80 & 70 & 60 & 50 & 40 & 30 & 23 \\
PMFS, mN & 76.1 & 76.6 & 76.8 & 76.8 & 76.9 & 76.9 & 77.1 & 77.3 \\
Thrust stand, mN & 76.3 & 77.1 & 77.6 & 77.9 & 77.9 & 78.4 & 79.2 & 79.2 \\
Difference, \% & 0.3 & 0.6 & 1.1 & 1.4 & 1.2 & 1.9 & 2.6 & 2.4 \\
Anode voltage, V & 300 & 300 & 300 & 300 & 300 & 300 & 300 & 300 \\
Xe ion energy, eV & 250 & 250 & 250 & 250 & 250 & 250 & 250 & 250 \\
Integrated sputter yield, atom/ion & 0.21 & 0.22 & 0.22 & 0.23 & 0.25 & 0.28 & 0.30 & 0.40 \\
Sputtered energy of carbon, eV & 2.0 & 2.1 & 2.2 & 2.2 & 2.5 & 2.5 & 2.7 & 3.1 \\
Momentum increase, \% & 0.6 & 0.6 & 0.6 & 0.7 & 0.8 & 0.8 & 0.9 & 1.3 \\
\hline
\end{tabular}

Table 5 Carbon sputter yield, average sputtered $\mathrm{C}$ atom energy, and the associated momentum increase as a function of incident $\mathrm{Xe}$ angle and energy for various thruster anode and gas flow settings

\begin{tabular}{lccccccc}
\hline \hline Thruster power, $\mathrm{kW}$ & 0.71 & 1.50 & 1.58 & 2.49 & 3.15 & 4.38 & 7.65 \\
PMFS, mN & 32.6 & 72.7 & 89.2 & 138.2 & 149.7 & 231.4 & 350.7 \\
Thrust stand, mN & 33.9 & 73.6 & 85.9 & 136.6 & 158.8 & 237.1 & 355.8 \\
Difference, \% & 3.6 & 1.2 & 3.8 & 1.2 & 5.7 & 2.4 & 1.4 \\
Anode voltage, V & 150 & 300 & 150 & 150 & 300 & 300 & 500 \\
Xe ion energy, eV & 130 & 250 & 130 & 130 & 250 & 250 & 440 \\
Integrated sputter yield, atom/ion & 0.04 & 0.18 & 0.04 & 0.04 & 0.18 & 0.18 & 0.44 \\
Sputtered energy of carbon, eV & 1.4 & 2.1 & 1.4 & 1.4 & 2.1 & 2.1 & 2.9 \\
Momentum increase, \% & 0.1 & 0.4 & 0.1 & 0.1 & 0.4 & 0.4 & 1.0 \\
\hline \hline
\end{tabular}




\section{B. Neutral Reflection and Charge Exchange}

A small fraction of the particles from the P5 HET hit the PMFS target as cold, unaccelerated neutrals. A cold neutral particle would bounce off the target, thus contributing twice the amount of momentum that it carries toward the PMFS target. However, these cold gas particles carry negligible momentum compared with the accelerated ions and, in this case, no neutral gas corrections were needed. Furthermore, the fraction of unaccelerated neutrals to accelerated ions is approximately $20 \%$ [16]. A more serious concern is hot neutrals created from fast ions undergoing charge exchange (CEX) with the atoms of an ambient neutral gas population. Typically, these energetic neutrals would go undetected by the Faraday probe that was used to detect the radial ion flux. However, as long as the charge-exchange fraction is low compared with the ion population and/or the concentration of charge-exchanged neutrals is proportional to the ion flux, charge-exchanged neutrals do not significantly affect the accuracy of the PMFS device. As estimated by Randolph et al. [13], only $2 \%$ of $200 \mathrm{eV} \mathrm{Xe} \mathrm{ions} \mathrm{undergo} \mathrm{CEX}$ within $100 \mathrm{~cm}$ with a background Xe pressure of $3 \times 10^{-6}$ torr. With all of these factors combined, the cold gas contributes negligible momentum compared with the accelerated ions in the P5 HET at nominal operating conditions.

The Xe ions that become implanted within the graphite target may eventually make their way back to the front surface and escape from the target as neutral Xe atoms. However, the neutral Xe atoms would escape in an isotropic distribution as cold neutrals and also carry away negligible momentum compared with the high-energy-incident Xe ions.

The effect of CEX particles and doubly charged ions is also negligible as long as the CEX and doubly ionized fluxes are directly proportional to the ion flux or the fraction of these CEX neutrals and doubly charged ions is small compared with singly charged ions. This is a reasonable assumption based on previous data obtained from the P5 HET [17].

\section{Conclusions}

The PMFS was shown to agree well with an inverted pendulum thrust stand, with a maximum observed difference of approximately $6 \%$ and an average difference of approximately $2 \%$ over a large range of force, ion energy, and neutral gas flow settings. It is often cumbersome or impossible to mount heavy thrusters on pendulumtype thrust stands. Likewise, the cost associated with designing an appropriate thrust stand can be prohibitive to some research efforts. As a viable alternative, the PMFS can be used as a highly accurate force sensor for a large range of flowing plasma conditions. Because the PMFS has demonstrated its accuracy as a plasma force diagnostic and is a simple, robust, and low-cost device, it should find considerable application in the flowing plasma and electric propulsion communities.

\section{Acknowledgments}

The authors would like to thank the University of Houston Institute for Space Systems Operations postdoctoral fellowship program and the Ad Astra Rocket Company for travel and experiment support.

\section{References}

[1] Chavers, D. G., and Chang-Díaz, F. R., "Momentum Flux Measuring Instrument for Neutral and Charged Particle Flows," Review of Scientific Instruments, Vol. 73, No. 10, 2002, pp. 3500-3507. doi: $10.1063 / 1.1505107$

[2] Cohen, S. A., Zonca, F., Timberlake, J., Bennett, T., Cuthbertson, J., Langer, W., and Motley, R., "An Instrument for Measuring the Momentum Flux From Atomic and Charged Particle Jets," Review of Scientific Instruments, Vol. 61, No. 11, 1990, pp. 3586-3591. doi:10.1063/1.1141575

[3] Nedzelskiy, I. S., Silva, C., Fernandes, H., Duarte, P., and Varandas, C. A. F., "Compact Cantilever Force Probe for Plasma Pressure Measurements," Review of Scientific Instruments, Vol. 78, No. 12, 2007, pp. 123505-123505-6. doi:10.1063/1.2813897

[4] Yeha, F. B., and Wei, P. S., "The Effect of Sheath on Plasma Momentum Transport to an Electrically Biased Surface," International Journal of Heat and Mass Transfer, Vol. 48, No. 11, May 2005, pp. 2198-2208. doi:10.1016/j.ijheatmasstransfer.2004.12.029

[5] Gnizdor, R. Y., Kozubsky, K. N., Koryakin, A. I., Lysikov, Y. M. Nesterenko, A. N., and Pridannikov S. Y., "Research of the Dynamic Pressure Distribution in the Plume of the SPT," 25th International Electric Propulsion Conference, Paper 1997-153, Aug. 1997.

[6] Haas, J. M., "Low-perturbation Interrogation of the Internal and Nearfield Plasma Structure of a Hall Thruster Using a High-Speed Probe Positioning System," Ph.D. Thesis, Dept. of Aerospace Engineering, Univ. of Michigan, Dearborn, MI, 2001.

[7] Haas, J. M., and Gallimore, A. D., "An Investigation of Internal Ion Number Density and Electron Temperature Profiles in a LaboratoryModel Hall Thruster," AIAA Paper 2000-3422, July 2000.

[8] Haas, J. M., Gulczinski, F. S., III., Gallimore , A. D., Spanjers, G. G., and Spores, R. A., "Performance characteristics of a $5 \mathrm{~kW}$ laboratory Hall thruster," AIAA Paper 1998-3503, July 1998.

[9] Hofer, R. R., "Development and Characterization of High-Efficiency, High-Specific Impulse Xenon Hall Thrusters," Ph.D. Dissertation, University of Michigan, Dearborn, MI, 2004.

[10] Haag, T. W., "Design of a Thrust Stand for High Power Electric Propulsion Devices," AIAA Paper 1989-2829, July 1989.

[11] Gulczinksi, F. S., Hofer, R. R., and Gallimore, A. D., "Near-Field Ion Energy and Species Measurements of a $5 \mathrm{~kW}$ Laboratory Hall Thruster," AIAA Paper 1999-2430, June 1999.

[12] Ziegler, J. F., and Biersack, J. P., SRIM: The Stopping and Range of Ions in Matter, Software Package, SRIM Ver., 2008.

[13] Randolph, T., Kim, V., Kaufman, H. R., Kozubsky, K., Zhurin, V. V., and Day, M., "Facility Effects on Stationary Plasma Thruster Testing," 23rd International Electric Propulsion Conference, Paper 1993-093, Sept. 1993.

[14] Stangeby, P. C., The Plasma Boundary of Magnetic Fusion Devices, Institute of Physics, Bristol, England, U.K., 2000, pp. 46, 113, 647.

[15] Doerner, R. P., Whyte, D. G., and Goebel, D. M., "Sputtering Yield Measurements During Low Energy Xenon Plasma Bombardment," Journal of Applied Physics, Vol. 93, No. 9, May 2003, pp. 5816-5823. doi:10.1063/1.1566474

[16] Hofer, R. R., Jankovsky, R. S., and Gallimore, A. D., "High-Specific Impulse Hall Thrusters, Part 2: Efficiency Analysis," Journal of Propulsion and Power, Vol. 22, No. 4, 2006, pp. 732-740. doi: $10.2514 / 1.15954$

[17] Azziz, Y., "Experimental and Theoretical Characterization of a Hall Thruster Plume," Ph.D. Dissertation, Aeronautics and Astronautics Dept., Massachusetts Institute of Technology, Cambridge, MA, 2007.

R. Myers

Associate Editor 